\title{
On Information Theoretic Interference Games With More Than Two Users
}

\author{
Randall A. Berry and Suvarup Saha \\ Dept. of EECS \\ Northwestern University \\ e-mail: rberry@eecs.northwestern.edu \\ suvarups@u.northwestern. edu
}

\begin{abstract}
We consider a model for information theoretic games on interference channels introduced in previous work. The players in these games are the users, who autonomously select their encoding and decoding strategy with the objective of maximizing their own rate. In previous work, a Nash equilibrium region for this game was defined and completely characterized for two-user linear deterministic interference channels. In particular for such channels it was shown that there always exists an efficient Nash equilibrium. In this paper, we consider extending this to models with more than two users. We show that some of the analysis extends to networks with more than two users, however, significant differences also emerge. For example, it may no longer be the case that there are always efficient equilibria.
\end{abstract}

\section{INTRODUCTION}

Mitigating interference is a key challenge in wireless networks (as well as some wireline cases). In an open environment, where the users (or device manufactures) have freedom in their choice of operating parameters, it is important to understand how the incentives of different users effect their desire to control interference. Such questions are naturally studied using game theory.

Here, we focus on a basic example of such a problem, namely a game among a set of users sharing an interference channel. In this channel each user communicates an independent message over a point-to-point link, and the links interfere with each other through cross-talk. Each user's objective is to maximize his own throughput; however, doing this effects the performance of the other users as well. The canonical information theoretic model for an interference channel is the Gaussian interference channel. The capacity region of this channel is not known in general. However, recently the capacity region for the two-user interference channel has been characterized to within one bit for all values of channel parameters [1]. Furthermore, it is shown in [2] that the high SNR behavior of the two-user Gaussian interference channel is in fact captured by a deterministic interference channel, for which the capacity region can be computed exactly using the results in [4]. (This type of deterministic model was first proposed in [5] for Gaussian relay networks.)

In [8], a game theoretic model for two users communicating over a general interference channel was developed. In particu-

This work was supported in part by NSF grants CNS-0905407 and CCF0905224 and by the Darpa ITMANET program under the grant W911NF-07$1-0028$. lar, the Nash equilibria region of the channel was defined as a natural extension of the information theoretic capacity region. If a pair of rates lie in this region then for long enough block lengths there exists a pair of encoding and decoding strategies from which neither user is willing to deviate if they require arbitrarily small probability of error. In [8], this region was then completely characterized for the two-user deterministic interference channel model from [2]. This region had a very simple structure and was always given by the intersection of a "box" with the capacity region. Moreover, it was shown that there are always Nash equilibria which are efficient, i.e., which lie on the maximum sum-rate boundary of the capacity region. In [9], these results were partially extended to the two-user Gaussian interference channel.

In this paper, we consider extending the game theoretic model in [8] to interference channels with more than two users. As in [8] we restrict our attention to the linear deterministic channel models. The analysis in [8] benefited from the fact the for two users, the capacity region of the deterministic channel is known. This is not the case for a general deterministic interference channel with more than two users. Hence, we focus on a few special cases $K$-user deterministic interference channels and use these to show that in some cases the insights from the two user case directly generalize, while in other cases they do not. Moreover, we show that with more than three users there will not always be an efficient Nash equilibria.

Other game theoretic approaches for interference channels have been studied before, mainly focusing on Gaussian models, e.g. [6], [7]. However, there are two key assumptions in these works: 1) the class of encoding strategies are constrained to use random Gaussian codebooks; 2) the decoders are restricted to treat the interference as Gaussian noise and are hence sub-optimal. Because of these restrictions, the formulation in these works are not information-theoretic in nature. For example, a Nash equilibrium found under these assumptions may no longer be an equilibrium if users can adopt a different encoding or decoding strategy as we allow here.

\section{Problem Formulation}

To begin, we define the basic interference channel game for $K$ users; this directly generalizes the $K=2$ user game presented in [8]. Communication starts at time 0. Each user $i=1, \ldots, K$ communicates by coding over blocks of 
length $N_{i}$ symbols. Transmitter $i$ sends on block $k$ information bits $b_{i 1}^{(k)}, \ldots, b_{i, L_{i}}^{(k)}$ by transmitting a codeword denoted by $\mathbf{x}_{i}^{(k)}=\left[\mathbf{x}_{i}^{(k)}(1), \ldots, \mathbf{x}_{i}^{(k)}\left(N_{i}\right)\right]$. All the information bits are equally probable and independent of each other. Receiver $i$ observes on each block $k$ an output sequence $\mathbf{y}_{i}^{(k)}=\left[\mathbf{y}_{i}^{(k)}(1), \ldots, \mathbf{y}_{i}^{(k)}\left(N_{i}\right)\right]$ through the interference channel, which specifies a stochastic mapping from the input sequences of users $1, \ldots, K$ to the output sequences of the users. Given the observed sequences up to block $k,\left\{\mathbf{y}_{i}^{(m)}\right\}_{m=1}^{k}$, receiver $i$ generates a guess $\hat{b}_{i \ell}^{(k)}$ for each information bit. Without loss of generality, we assume that this is done via maximum-likelihood decoding on each bit.

Note that this communication scenario is more general than the one usually used in multiuser information theory, as we allow the users to code over different block lengths. Such generality is necessary here, since even though the users may agree $a$ priori on a common block length, a selfish user may unilaterally decide to choose a different block length during the actual communication process.

A strategy $s_{i}$ of user $i$ is defined by its message encoding, which we assume to be the same on every block and involves:

- the number of information bits $L_{i}$ and the block length $N_{i}$ of the codewords,

- the codebook $\mathcal{C}_{i}$ employed by transmitter $i$,

- the encoder $f_{i}:\left\{1, \ldots, 2^{L_{i}}\right\} \times \Omega_{i} \rightarrow \mathcal{C}_{i}$, that maps on each block $k$ the message $m_{i}^{(k)}:=\left(b_{i 1}^{(k)}, \ldots b_{i, L_{i}}^{(k)}\right)$ to a transmitted codeword $\mathbf{x}_{i}^{(k)}=f_{i}\left(m_{i}^{(k)}, \omega_{i}^{(k)}\right) \in \mathcal{C}_{i}$,

- the rate of the code, $R_{i}\left(s_{i}\right)=L_{i} / N_{i}$.

Let $\mathbf{s}=\left(s_{1}, \ldots, s_{K}\right)$ denote a strategy profile, i.e., a choice of strategies for each user $i=1, \ldots, K$. A given profile $\mathbf{s}$ jointly determines the probabilities of error $p_{i}^{(k)}:=$ $\frac{1}{L} \sum_{\ell=1}^{L_{i}} \mathcal{P}\left(\hat{b}_{i \ell}^{(k)} \neq b_{i \ell}^{(k)}\right), i=1, \ldots, K$. Note that if the users use different block lengths, the error probability could vary from block to block even though each user uses the same encoding for all the blocks.

The encoder of each transmitter $i$ may employ a stochastic mapping from the message to the transmitted codeword; $\omega_{i}^{(k)} \in \Omega_{i}$ represents the randomness in that mapping. We assume that this randomness is independent between the two transmitters and across different blocks and is only known at the respective transmitter and not at any of the receivers.

For a given error probability threshold $\epsilon>0$, we define an $\epsilon$-interference channel game as follows. Each user $i$ chooses a strategy $s_{i}$ and receives a pay-off of $\pi_{i}(\mathbf{s})=R\left(s_{i}\right)$ if $p_{i}^{(k)}(\mathbf{s}) \leq \epsilon$, for all $k$; otherwise, $\pi_{i}(\mathbf{s})=0$. In other words, a user's pay-off is equal to the rate of the code provided that the probability of error is no greater than $\epsilon$. A strategy profile $\mathbf{S}$ is defined to be $(1-\epsilon)$-reliable provided that it results in an error probability $p_{i}^{k}(\mathbf{s})$ of less than $\epsilon$ for $i=1, \ldots, K$ and all $k$.

For an $\epsilon$-game, a strategy profile $\mathbf{s}^{*}=\left(s_{1}^{*}, \ldots, s_{K}^{*}\right)$ is a Nash equilibrium (NE) if no single user can unilaterally deviate and improve his pay-off, i.e. if for each user $i=1, \ldots, K$, there is no other strategy $s_{i}$ such that ${ }^{1}$ $\pi_{i}\left(s_{i}, \mathbf{s}_{-i}^{*}\right)>\pi_{i}\left(s_{i}^{*}, \mathbf{s}_{-i}^{*}\right)$. If user $i$ attempts to transmit at a higher rate than what he is receiving in a NE and the other users do not change their strategy, then user $i$ 's error probability must be greater than $\epsilon$. Similarly, a strategy profile $\mathbf{s}^{*}$ ) is an $\eta$-Nash equilibrium ${ }^{2}$ ( $\eta$-NE) of an $\epsilon$-game if no user can unilaterally deviate and improve his pay-off by more than $\eta$, i.e. if for each user $i$, there is no other strategy $s_{i}$ such that $\pi_{i}\left(s_{i}, \mathbf{s}_{-i}^{*}\right)>\pi_{i}\left(s_{i}^{*}, \mathbf{s}_{-i}^{*}\right)+\eta$. Note that when a user deviates, it does not care about the reliability of the other users but only its own reliability. So in the above definitions $\left(s_{i}, \mathbf{s}_{-i}^{*}\right)$ is not necessarily $(1-\epsilon)$-reliable.

Given any $\bar{\epsilon}>0$, the capacity region $\mathcal{C}$ of the interference channel is the closure of the set of all rate vectors $\left(R_{1}, \ldots, R_{K}\right)$ such that for every $\epsilon \in(0, \bar{\epsilon})$, there exists a $(1-\epsilon)$-reliable strategy profile $\mathbf{s}$ that achieves the rate vector $\left(R_{1}, \ldots, R_{K}\right)$. The Nash equilibrium region $\mathcal{C}_{\mathrm{NE}}$ of the interference channel is the closure of the set of rate pairs $\left(R_{1}, \ldots, R_{K}\right)$ such that for every $\eta>0$, there exists a $\bar{\epsilon}>0$ (dependent on $\eta$ ) so that if $\epsilon \in(0, \bar{\epsilon})$, there exists a $(1-\epsilon)$-reliable strategy profile $\mathbf{s}$ that achieves the rate vector $\left(R_{1}, \ldots, R_{K}\right)$ and is a $\eta$-NE. Clearly, $\mathcal{C}_{\mathrm{NE}} \subseteq \mathcal{C}$. Here, our goal is to characterize which rate points in $\mathcal{C}$ are included in $\mathcal{C}_{\mathrm{NE}}$.

Following [8], we consider this question in the context of the linear deterministic interference channel model introduced in [2]. In this channel, channel input for each is interpreted as a succession of levels: $x=0 . b_{1} b_{2} b_{3} b_{4} b_{5} \ldots$ each representing one bit of the real-valued input to the corresponding Gaussian channel. The most significant bit coincides with the highest level, the least significant bit with the lowest level. Noise is modeled by truncation. Bits of smaller order than the noise are lost.The signal from transmitter $i$, as observed at receiver $j$, is scaled by a nonnegative integer gain $a_{j i}=2^{n_{j i}}$ (equivalently, the input column vector is shifted up by $n_{j i}$ ). At each time $t$, the input and output, respectively, at link $i$ are $\mathbf{x}_{i}(t), \mathbf{y}_{i}(t) \in\{0,1\}^{q}$, where $q=\max _{i j} n_{i j}$. Note that $n_{i i}$ corresponds to $\log _{2} \mathrm{SNR}_{i}$ and $n_{j i}$ corresponds to $\log _{2} \mathrm{INR}_{j i}$, where $\mathrm{SNR}_{i}$ is the signal-to-noise ratio of link $i$ and $\mathrm{INR}_{j i}$ is the interference-to-noise ratio at receiver $j$ from transmitter $i$ in the corresponding Gaussian interference channel. To model the super-position of signals at each receiver, the bits received on each level are added modulo two. The channel output at receiver $i$ is then given by

$$
\mathbf{y}_{i}(t)=\sum_{j=1}^{K} \mathbf{S}^{q-n_{i j}} \mathbf{x}_{j}(t),
$$

where summation and multiplication are in the binary field and $\mathbf{S}$ is a $q \times q$ shift matrix (e.g. see [5]).

In [8], $\mathcal{C}_{\mathrm{NE}}$ was completely characterized for the linear deterministic channel model with $K=2$ users. In particular,

\footnotetext{
${ }^{1}$ We use the notation $\mathbf{s}_{-i}$ to denote the set of strategy choices for every user except user $i$. Also, with a slight abuse of notation we use $\left(s_{i}, \mathbf{s}_{-i}\right)$ to denote the profile $\mathbf{s}$.

${ }^{2}$ In the game theoretic literature, this is often referred to as an $\epsilon$-Nash equilibrium or simply an $\epsilon$-equilibrium for a game [10, page 143].
} 


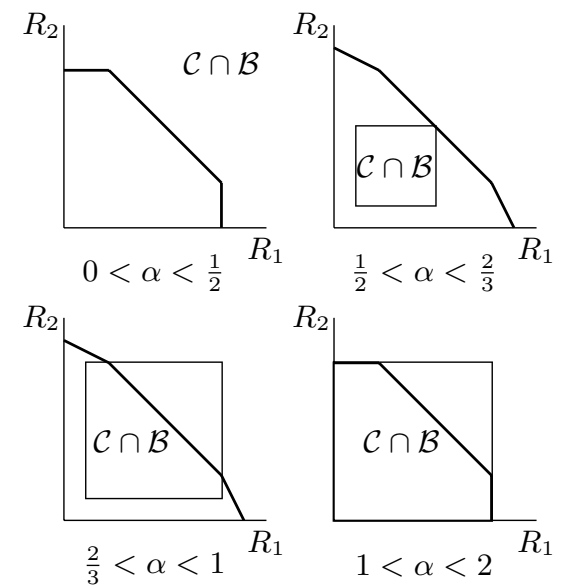

Fig. 1. Examples of $\mathcal{C}_{\mathrm{NE}}$ for for a symmetric deterministic interference channel with normalized cross gain $\alpha$.

$\mathcal{C}_{\mathrm{NE}}$ was shown to be equal to the intersection of a "box" $\mathcal{B}$ and the capacity region $\mathcal{C}$ of the deterministic channel (see Figure 1). The intersection is always non-empty and contains at least one point on the sum-rate boundary of $\mathcal{C}$.

\section{ANALYSIS}

\section{A. Bounds}

In this section, we generalize the "box" bounds in [8] to a $K$-user linear deterministic interference channel. We first make some preliminary definitions. For a given user $i$, let $L_{i}=\left(n_{i i}-\max _{j} n_{i j}\right)^{+}$denote the number of interference free levels at user $i$ 's receiver; at these levels user $i$ never sees any interference from another user. Let $\mathcal{D}_{i}$ denote the $n_{i i}$ direct levels at user $i$ 's receiver, i.e. the levels that are connected to that user's transmitter. Let $\mathcal{Q}_{i}$ be the set of levels at user $i$ 's receiver that are connected to an interference free level for any other user $j \neq i$. Finally, let $U_{i}=\left|\mathcal{D}_{i} \backslash \mathcal{Q}_{i}\right|$; this is the number of direct levels at user $i$ that are not interfered with by any other user's interference free levels. The generalization of the bounds in [8] is then given in the following lemma.

Lemma 1: If $\left(R_{1}, \ldots, R_{K}\right) \in \mathcal{C}_{\mathrm{NE}}$, then for each user $i$, $L_{i} \leq R_{i} \leq U_{i}$.

Let $\mathcal{B}=\prod_{i}\left[L_{i}, U_{i}\right]$ be a $K$-dimensional box formed by taking the Cartesian product of these bounds. This lemma can then be re-stated as saying that $\mathcal{C}_{\mathrm{NE}} \subseteq \mathcal{C} \cap \mathcal{B}$, i.e. the Nash equilibrium region must lie within the intersection of the capacity region and this box. The proof of Lemma 1 follows from a similar argument as in the two-user case; we briefly provide some intuition behind this. It can not be that a user achieves a rate lower than $L_{i}$ in a Nash equilibrium since that user can always change his strategy to simply transmitting uncoded information on the $L_{i}$ interference free levels and so improve his pay-off to at least $L_{i}$. Furthermore, when all users are sending at maximum rate on their $L_{j}$ interference free levels, this will create maximum entropy interference to each user $i$ on $\left|\mathcal{Q}_{i}\right|$ levels rendering them unusable. This leaves $U_{i}$ remaining levels, which upper bounds a user's reliable rate for $\epsilon$ small enough.
Following [8], we define a profile of strategies $\left(s_{1}, \ldots, s_{K}\right)$ to be a partial Bernoulli profile if: (1) each user $i$ sees i.i.d. Bernoulli-1/2 interference on $k_{i}$ levels at his receiver, which interfere with his transmitted signal and (2) each user $i$, transmits at rate $R_{i}=n_{i i}-k_{i}$ with zero probability of error. As the next lemma states, such strategies are useful for showing that a given rate point is in $\mathcal{C}_{\mathrm{NE}}$.

Lemma 2: If there exist a partial Bernoulli profile that achieves the rate point $\left(R_{1}, \ldots, R_{K}\right)$, then $\left(R_{1}, \ldots, R_{K}\right) \in$ $\mathcal{C}_{\mathrm{NE}}$.

Using this, we then have the following result which states that the "lower" corner of $\mathcal{B}$ is always in $\mathcal{C}_{N E}$.

Lemma 3: The rate point $\left(L_{1}, \ldots, L_{K}\right)$ are always in $\mathcal{C}_{N E}$.

This follows as in the two-user case by considering a strategy profile in which each user transmits uncoded information on his interference free levels and i.i.d. Bernouli-1/2 noise on the remaining levels that appear at any receiver. Note that if $n_{i j}>n_{j j}$ then this requires user $j$ to transmit i.i.d. noise at some levels that appear below the noise floor at that user's own receiver. It can be seen that this satisfies our requirements for a partial Bernoulli profile.

\section{B. One-to-Many and Many-to-One channels}

Two classes of deterministic interference channels for which the capacity region is known for more than two users are the one-to-many and many-to-one models studied in [3]. For the many-to-one interference channel, interference only occurs at one receiver, which without loss of generality we assume to be 1 . For the one-to-many case, only transmitter 1 causes interference to any other receiver.

The next lemma shows that in the one-to-many case, the bounds in Lemma 1 have a relatively simple form.

Lemma 4: In a one-to-many deterministic interference channel, $U_{1}=L_{1}=n_{11}$, and for each user $i \neq 1$, $U_{i}=L_{i}+\min \left(n_{i i},\left(n_{i 1}-n_{11}\right)^{+}\right)$.

Proof: Since user 1 does not see any interference, it must be that $L_{1}=U_{1}=n_{11}$.

Now suppose that $n_{i 1} \leq n_{11}$ for some user $i \neq 1$. In this case, if user $i$ can receive any interference on a level, it must be receiving interference from one of user 1's interference free levels. Thus, it follows that $U_{j}=L_{j}$, which is the same as the conclusion of the lemma for $n_{i 1} \leq n_{11}$.

If instead, $n_{i 1} \geq n_{11}$ then when user 1 transmits only on his $n_{11}$ interference free levels, the $\left(n_{i 1}-n_{11}\right)$ lowest levels at receiver $i$ will not see any interference. Adding the max of these and $n_{i i}$ to user $i$ 's $L_{i}$ interference free levels yields the desired expression for $U_{i}$.

Using this we then have the following partial characterization of $\mathcal{C}_{\mathrm{NE}}$ for a one-to-many channel.

Proposition 1: In a one-to-many deterministic interference channel, if $n_{i 1} \leq n_{11}$ for all $i$, then $\mathcal{C}_{\mathrm{NE}}=\left(L_{1}, \ldots, L_{K}\right)$; otherwise $\mathcal{C}_{\mathrm{NE}}$ contains multiple points including $\left(U_{1}, \ldots, U_{K}\right)$.

Proof: If $n_{i 1} \leq n_{11}$ for all $i$, then the result follows directly from Lemmas 3 and 4 . If this condition is not satisfied, consider the strategy profile in which user 1 transmits uncoded information only on his interference free levels. Then each user 
$i \neq 1$ can send uncoded information on all of the $U_{1}$ levels. This is a partial Bernoulli profile and so from Lemma 2, we have that $\left(U_{1}, \ldots, U_{K}\right) \in \mathcal{C}_{\mathrm{NE}}$.

In a two user interference channel, $\mathcal{C}_{\mathrm{NE}}=\mathcal{B} \cap \mathcal{C}$. The preceding proposition shows that when $n_{i 1} \leq n_{11}$ for all $i$ this also the case in a one-to-many interference channel with more than two users. However, when $n_{i 1}>n_{11}$ for at least two users $i \neq 1$ this will no longer be true. To see this, consider a symmetric one-to-many channel with three users in which $n_{21}=n_{31}>n_{11}$. For such a channel the rate pair $\left(U_{1}, U_{2}, L_{3}\right)$ is in $\mathcal{B} \cap \mathcal{C}$. Indeed, this rate pair can be achieved (with zero probability of error) by modifying the strategy profile in the proof of Proposition 1 for achieving $\left(U_{1}, U_{2}, U_{3}\right)$ by having user 3 only transmit on its $L_{3}$ interference free levels. However, for $\eta<1$, this rate pair can not be a $\eta$-NE for $\epsilon$ small enough. This is because due to the symmetry any rate that user 2 is achieving can also be achieved by user 3 and since $U_{2}=U_{3} \geq L_{3}+1$, user 3 can deviate and improve his rate by 1 . The issue here is that the strategic interaction of user 1 with user 2 and 3 is coupled due to the structure of the channel. The constraints in the box do not account for this coupling. Taking this into account it can be seen that in a symmetric one-to-many channel, it must be that $\mathcal{C}_{\mathrm{NE}}$ is simply the line segment in $\mathbb{R}^{K}$ connecting the points $\left(L_{1}, \ldots, L_{K}\right)$ with $\left(U_{1}, \ldots, U_{K}\right)$. The next lemma generalizes this example:

Lemma 5: In a one-to-many deterministic interference channel with $K>2$ users, if $n_{i 1}>n_{11}$ for at least two users $i \neq 1$, then $\mathcal{C}_{\mathrm{NE}} \neq \mathcal{B} \cap \mathcal{C}$.

Another property in the two-user case is that $\mathcal{C}_{\mathrm{NE}}$ always contains an efficient rate point. Once again with more than two users in a one-to-many channel, this may no longer be true as summarized in the following Proposition.

Proposition 2: In a one-to-many deterministic interference channel with $K>2$ users, $\mathcal{C}_{\mathrm{NE}}$ will not contain a sum-rate optimal point unless $n_{i 1}>n_{i i}+n_{11}$ for all $i \neq 1$ except at most 1 user.

We know from Proposition 1 that $\left(U_{1}, \ldots, U_{K}\right) \in \mathcal{C}_{\mathrm{NE}}$ and from Lemma 1 it follows that this rate point dominates any other rate point in $\mathcal{C}_{\mathrm{NE}}$. Hence to prove the theorem we simply have to show that this rate point is not efficient. This can be done by using the characterization of the maximum sum-rate in [3]. Note that the condition that $n_{i 1}>n_{i i}+n_{11}$ means that user 1's creates very strong interference at user $i$. As an example, again consider a symmetric many to one channel with $K=3$ users and assume that $n_{11}=n_{22}=n_{33}=3$ and $n_{21}=n_{31}=2$. For this channel, $\left(U_{1}, U_{2}, U_{3}\right)=(3,1,1)$, resulting in a sum-rate of 5 . However, the maximum sumrate of this channel is 7 , which is achieved by the rate profile $\left(R_{1}, R_{2}, R_{3}\right)=(1,3,3)$. The issue here is that when user 1 uses one of its two most significant levels, it creates interference at two other users. Effectively a gain by user 1 causes a net reduction in the sum-rate, resulting in the inefficiency. To characterize this inefficiency, for a given channel define the efficiency

$$
\gamma=\frac{R_{\text {sum }}^{\mathcal{C}_{\mathrm{NE}}}}{R_{\text {sum }}^{\mathcal{C}}},
$$

where $R_{\text {sum }}^{\mathcal{C}_{\mathrm{NE}}}$ is maximum sum-rate in $\mathcal{C}_{\mathrm{NE}}$ and $R_{\text {sum }}^{\mathcal{C}}$ is the maximum sum-rate in $\mathcal{C}$ (e.g. in the previous example $\gamma=\frac{5}{7}$ ). It can then be seen that in a one-to-many interference channel with $K$ users $\gamma \geq \frac{1}{K-1}$, and this bound is achieved in a channel in which $n_{11}=n_{i 1}=n_{i i}$ for all $i$.

Next we consider the many-to-one case. By a similar argument as that used to prove Lemma 4 we have the following lemma for the many-to-one case. In this case, when $n_{1 i}>n_{i i}$ for some $i$ then in general there does not seem to be a simpler expression for $U_{1}$.

Lemma 6: In a many-to-one deterministic interference channel, $U_{i}=L_{i}=n_{i i}$ for all $i \neq 1$ and if $n_{1 i} \leq n_{i i}$ for all $i \neq 1$, then $U_{1}=L_{1}$.

In the many-to-one case, we can exactly characterize $\mathcal{C}_{\mathrm{NE}}$ as given next.

Proposition 3: In a many-to-one deterministic interference channel, $\mathcal{C}_{\mathrm{NE}}=\mathcal{B} \cap \mathcal{C}$.

Proof: When $n_{1 i} \leq n_{i i}$ for all $i \neq 1$, then Lemma 6 implies that $\mathcal{B}$ is the single point $\left(L_{1}, \ldots, L_{K}\right)$, which we know is in $\mathcal{C}_{\mathrm{NE}}$ from Lemma 3 . When this is not true, then $\mathcal{B}$ is a line segment connecting the points $\left(L_{1}, \ldots, L_{K}\right)$ to the point $\left(U_{1}, L_{2}, \ldots, L_{K}\right)$. To complete the proof, we need to show that the point $\left(U_{1}, L_{2}, \ldots, L_{K}\right)$ is also in $\mathcal{C}_{\mathrm{NE}}$. To do this consider the partial Bernoulli profile in which each user $i$ transmits only on his $n_{i i}$ interference free levels and user 1 transmits on each level for which he is not receiving interference.

In this case, $\mathcal{C}_{\mathrm{NE}}$ will always contain an efficient rate point as summarized next.

Proposition 4: In a many-to-one deterministic interference channel, $\mathcal{C}_{\mathrm{NE}}$ always contains a sum-rate optimal rate point.

This follows from again using the characterization of the sum-rate point in [3]. In this case it can be seen that this will be exactly equal to the sum-rate achieved by the point $\left(U_{1}, \ldots, U_{K}\right)$.

The previous results show that the results in [8] generalize directly to the many-to-one case, but not to the one-to-many case. A key difference between these two models is that in the many-to-one case, each users action effects at most one other user (user 1), while in the one-to-many case user 1's actions can effect multiple other users. This difference is at the heart of the different behavior we observe with respect to these two classes of channels.

\section{Three-user bi-symmetric models}

As another example we consider a class of three-user bi-symmetric channels defined as all three-user channels in which $n_{11}=n_{22}=n_{33}, n_{12}=n_{32}=n_{21}=n_{23}$ and $n_{13}=n_{31}=0$; i.e. there is no interference between users 1 and 3 and the interference between users 1 and 2 is the same as that between users 3 and 2 . Given such a channel, let $\mathcal{C}^{12}$ denote the capacity of the two user (symmetric) interference channel created by removing user 3 ; and let $\mathcal{C}^{23}$ denote the capacity of the channel created by removing user 1 . Note that the symmetry assumption implies that $\mathcal{C}^{12}=\mathcal{C}^{23}$ The 
next lemma shows that the symmetry of this channel can be exploited to characterize the optimal sum-rate.

Lemma 7: Given a three-user bi-symmetric channel, if the rate profile $\left(R_{1}, R_{2}\right) \in \mathcal{C}^{12}$, then $\left(R_{1}, R_{2}, R_{1}\right) \in \mathcal{C}$. Furthermore, if the rate profile $\left(R_{1}, R_{2}, R_{3}\right) \in \mathcal{C}$ is sum-rate optimal, then it must be that $R_{1}=R_{3}$ and $\left(R_{1}, R_{2}\right) \in \mathcal{C}^{12}$.

Proof: If $\left(R_{1}, R_{2}\right) \in \mathcal{C}$, then from [8] it follows that this rate pair can be achieved by either uncoded transmission or simply using repetition coding across two or more levels (and possibly time-sharing). Given such a coding scheme, consider the three-user channel in which users 1 and 2 implement the same scheme and user 3 simply copies the scheme of user 1 . Since user 3 does not interfere with user 1 , user 1 will still be able to achieve rate $R_{1}$ and from the symmetry, user 3 will also be able to achieve $R_{1}$. Next consider user 2 . If in the two-user case, user 2 was receiving Bernoulli-1/2 interference due to user 1 transmitting uncoded information on a level, it will still see the same interference due to the superposition of user 1 and user 3's signals on that level. Likewise, if in the two-user case, user 2 was receiving a repetition of user 1's signal on two levels, then in the three user channel it will be receiving a repetition of the superposition of user 1 and 3's signals on the same levels. Hence, user 2 can still achieve rate $R_{2}$, proving the first part of the lemma.

To prove the second part, suppose that $\left(R_{1}, R_{2}, R_{3}\right) \in \mathcal{C}$ is sum-rate optimal with $R_{1}>R_{3}$. It must be that $\left(R_{1}, R_{2}\right) \in$ $\mathcal{C}^{12}$ since the presence of user 3 can only decrease the sum-rate of these two users. But then the first part of the lemma implies that $\left(R_{1}, R_{2}, R_{1}\right) \in \mathcal{C}$, which would have a higher sum-rate than $\left(R_{1}, R_{2}, R_{3}\right)$, contradicting our initial assumption.

Next we turn to the Nash equilibria region for this channel. Let $\mathcal{C}_{\mathrm{NE}}^{12}$ and $\mathcal{C}_{\mathrm{NE}}^{23}$ denote the Nash equilibria regions for the corresponding two user channels. The next lemma relates these to $\mathcal{C}_{\mathrm{NE}}$ for the three-user channel.

Lemma 8: In a three user bi-symmetric channel, if $\left(R_{1}, R_{2}, R_{3}\right) \in \mathcal{C}_{\mathrm{NE}}$ then it must be that $\left(R_{1}, R_{2}\right) \in \mathcal{C}_{\mathrm{NE}}^{12}$ and $\left(R_{2}, R_{3}\right) \in \mathcal{C}_{\mathrm{NE}}^{23}$.

Proof: This follows from noting that the bounds $U_{i}$ and $L_{i}$ in Lemma 1 will be the same for each user $i$ in the three user channel and in the corresponding two user channel. Since in the two-user channel $\mathcal{C}_{\mathrm{NE}}=\mathcal{B} \cap \mathcal{C}$, it follows that if a achievable rate pair is not in $\mathcal{C}_{\mathrm{NE}}^{12}$, then it must lie outside of $\mathcal{B}$ for the two user channel and thus also be outside of $\mathcal{B}$ for the three user case. Hence it can not be a Nash equilibrium.

Let $\alpha=\frac{n_{21}}{n_{11}}$ denote the normalized cross-gain for each of the two-user interference channels. As shown in Figure 1, the structure of $\mathcal{C}_{\mathrm{NE}}$ in the two user case depends on the value of this parameter. The next proposition shows that this parameter determines the efficiency in the three user case.

Proposition 5: In a three-user bi-symmetric channel, $\mathcal{C}_{\mathrm{NE}}$ contains a sum-rate optimal point only when $\alpha \geq 2 / 3$.

Proof: From Lemma 7, it follows that the maximum sumrate is given by maximizing $2 R_{1}+R_{2}$ over all $\left(R_{1}, R_{2}\right) \in \mathcal{C}^{12}$. From the structure of the capacity region, it can be seen that for $\alpha<2 / 3$, the solution to this problem will not lie in $\mathcal{C}_{\mathrm{NE}}^{12}$ and so from Lemma 8 it can not be in $\mathcal{C}_{\mathrm{NE}}$. When $\alpha>2 / 3$, this point does lie in $\mathcal{C}_{\mathrm{NE}}^{12}$. This can be translated into an equilibrium strategy for the three user case by having users 1 and 2 use the same strategy and having user 3 copy user 1's strategy.

By a similar argument as in this proof, we can also determine the efficiency $\gamma$ for any channel in this family as a function of $\alpha$. This has the following form:

$$
\gamma= \begin{cases}\frac{3-3 \alpha}{3-2 \alpha}, & 0 \leq \alpha \leq 1 / 2, \\ \frac{3 \alpha}{2}, & 1 / 2 \leq \alpha \leq 2 / 3, \\ 1, & 2 / 3 \leq \alpha\end{cases}
$$

Hence, the minimum efficiency for this class of channels is $3 / 4$, which occurs for $\alpha=1 / 2$. Note that this is better than the worst-case efficiency of $\frac{1}{3}$ for a 3 user one-to-many channel. This suggests that having mutual interference is beneficial for incentivizing cooperative behavior.

We also note that for this class of channels, it can again be shown that in general $\mathcal{C}_{\mathrm{NE}}$ is not equal to $\mathcal{B} \cap \mathcal{C}$.

\section{Conclusions}

We have studied the Nash equilibria region from [8] for three classes of deterministic interference channels with more than 2 users: one-to-many channels, many-to-one channels and 3 user bi-symmetric channels. We have seen that the Nash equilibrium region behaves differently in each of these cases and in general may not contain an efficient point. Characterizing these regions and the efficiency for more general $M$-user channels is left to future work as is relating these results to the corresponding Gaussian channel models.

\section{REFERENCES}

[1] R. Etkin, D. Tse, and H. Wang, "Gaussian Interference Channel Capacity to within One Bit," IEEE Trans. on Information Theory, Vol. 54, No. 12, Dec. 2008.

[2] G. Bresler and D. Tse, "The Two-User Gaussian Interference Channel: A Deterministic View," European Transactions in Telecommunications, vol. 19, pp. 333-354, April 2008.

[3] G. Bresler, A. Parekh, and D. Tse, "The Approximate Capacity of the many-to-one and one-to-many Gaussian interference channel," Allerton Conference on Communication, Control and Computing, Monticello, IL, Sept. 2007.

[4] A. El Gamal and M. Costa, "The Capacity Region of a Class of Deterministic Interference Channels," IEEE Transactions on Information Theory, Vol. IT-28, No. 2, pp. 343-346, March 1982.

[5] S. Avestimehr, S. Diggavi, and D. Tse, "Wireless Network Information Flow," Allerton Conference on Communication, Control, and Computing, Monticello, IL, September 2007.

[6] R. Etkin, A. P. Parekh and D.Tse, "Spectrum Sharing in Unlicensed Bands", IEEE Journal on Selected Areas of Communication, vol. 25, no. 3, pp. 517-528, April 2007.

[7] S. T. Chung, S. J. Kim, J. Lee, and J.M. Cioffi, "A game-theoretic approach to power allocation in frequency-selective Gaussian interference channels," Proceedings of IEEE ISIT, pp. 316-316, June 2003.

[8] R. Berry and D. Tse, "Information Theoretic Games on Interference Channels," Proceeding of IEEE ISIT, pp. 2518-2522, July 2008.

[9] R. Berry and D. Tse, "Information Theory Meets Game Theory on the Interference Channel," Proceedings of IEEE Information Theory Workshop, Volos, Greece, pp. 140-144, June 10-12, 2009.

[10] R. Myserson, Game Theory: Analysis of conflict, Harvard University Press, Cambridge MA, 1991. 